\title{
Interference of Simulated IEEE 802.11 Links with Directional Antennas
}

\author{
Michael Rademacher* and Karl Jonas ${ }^{\dagger}$ \\ Department of Computer Science, Bonn-Rhein-Sieg University of Applied Sciences \\ Sankt Augustin, Germany \\ Email: *michael.rademacher@h-brs.de, ${ }^{\dagger}$ karl.jonas@h-brs.de
}

\begin{abstract}
WiFi-based Long Distance (WiLD) networks have emerged as a promising alternative approach for Internet in rural areas. The main hardware components of these networks are commercial off-the-shelf $\mathrm{WiFi}$ radios and directional antennas. During our experiences with real-world WiLD networks, we encountered that interference among long-distance links is a major issue even with high gain directional antennas. In this work, we are providing an in-depth analysis of these interference effects by conducting simulations in ns-3. To closely match the real-world interference effects, we implemented a module to load radiation pattern of commonly used antennas. We analyze two different interference scenarios typically present as a part of larger networks. The results show that side-lobes of directional antennas significantly influence the throughput of long-distance WiFi links depending on the orientation. This work emphasizes that the usage of simple directional antenna models needs to be considered carefully.
\end{abstract}

Index Terms-WiFi-based Long Distance (WiLD); interference; directional antennas

\section{INTRODUCTION AND MOTIVATION}

A major problem for rural areas is inaccessibility to affordable broadband Internet connections. This may force companies and people into a difficult decision: whether to leave a specific region or stay behind and hope for better connectivity. To decrease the costs of broadband in rural regions, various alternatives are evaluated to establish high-bandwidth connections. One of these alternatives are WiFi-based Long Distance (WiLD) networks. Given their distribution in the consumer sector, WiFi radios are well developed, sold at a decent charge, have a low energy consumption and perform solidly in the license free Industrial, Scientific and Medical (ISM) or Unlicensed National Information Infrastructure (U-NII) band [1]. WiLD networks consist of multiple wireless links typically between 1 and $25 \mathrm{~km}$ using high-gain directional antennas in a Multi-Radio Multi-Channel Wireless Mesh Network (MR-MC WMN) topology. The majority of WiLD deployments are operated by so-called Wireless Internet Service Providers (WISPs) around the globe and numerous vendors provide commercial WiLD hardware [2], [3], [4]. In 2015, the Wireless Internet Service Provider Assocication (WISPA) estimated that 3000 different WISPs were operating in the USA.

One key issue of all radio networks, and therefore of WiLD networks alike, is interference. The usage of frequencies needs to be coordinated among the participants. In the unlicensed bands, several participants compete for the available spectrum following rules defined by the MAC protocol. An example of such rules is the so called Distributed Coordination Function (DCF) specified in the IEEE 802.11 standard [5]. There are several other possibilities to avoid interference like networkwide Channel Assignment (CA), Transmission Power Control (TPC), rate adaption and directional antennas [6].

In this work, we investigate interference among WiFi radios operating on the same channel. Our specific contribution is the usage of directional antennas, including a small extension to ns-3 [7]. While our goal is to understand interference effects in larger networks, we start with small and isolated parts of a WiLD network for better illustration of the various effects.

The rest of this paper is structured as follows. In Section II we shortly summarize previous work. Section III presents our methodology. Section IV is the main part of this work and presents the conducted simulations. This work closes with a conclusion and future work items in Section V.

\section{RELATED WORK}

The author in [8] identifies three different types of interference in a mesh topology which we visualize in Figure 1. Intra-flow interference occurs when a node uses a channel to communicate with multiple nodes using multiple radios. Inter-flow interferences occur between two links from different paths but in the same interference range. External inferences occur due the transmission of other participants using the same frequency. The author identifies these three interference types assuming omni-directional antennas. In this work, we will investigate in detail the first two types with directional antennas.

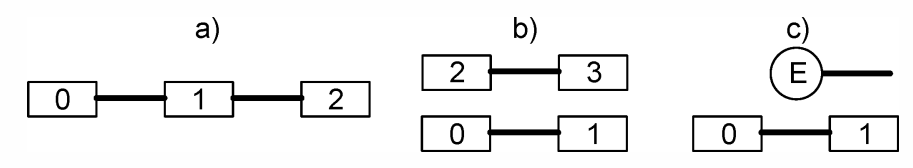

Fig. 1: Different types of interference: a) Intra-flow, b) Interflow, c) external interference. Figure schematically redrawn from [8].

The authors in [9] propose a MAC-protocol called DMAC designed for multi-hop ad-hoc networks that exploits directional antennas by distributing RTS packets. The authors consider beam-forming antennas for their protocol. In addition, they were the first to identify challenges which specifically 
occur with directional antennas: deafness and directional hidden terminals. Since then, multiple researchers [10], [11], [12] have elaborated on these challenges and we refer the reader to [6] for a comprehensive description. All mentioned publications motivate their research with mobile ad-hoc networks in combination with either steerable or beamforming antennas. Our described use-case of WiLD networks is different. Nodes are rather stationary and antennas are usually pointed based on the desired topology with significant distance in between. Side-lobes are not considered in previous publications but play a vital role in our investigations.

For a joint simulation of $\mathrm{WiFi}$ and directional antennas, the authors in [10], [11] use the QualNet and the authors in [12] use the OPNET simulator. A short overview of simulators using directional wireless communication is provided in [13].

The authors in [14] conducted outdoor measurements to prospect the relationship of directional antennas and adjacent channel interference. The authors conducted test with $250 \mathrm{~m}$ links using two directional antennas which are separated between 1.5 and $3 \mathrm{~m}$ attached to independent radios on each site. This setup corresponds to the inter-flow interference case. Despite the high directivity of the antennas, the authors found at least $40 \mathrm{MHz}$ separation is needed to provide independent throughput on both interfaces, otherwise, an increasing Packet Error Rate (PER) or the DCF decreases the throughput. However, they did not provide insights for the origin of the interference. The authors in [15] describe interference measurement in a IEEE 802.11a based metropolitan multi-radio test-bed with links distance between 1 and $5 \mathrm{~km}$. The authors emphasize the importance of a CA algorithm due to inter-flow and external interference even when using highly directional antennas. The authors in [16] conducted measurements using omni-antennas in a semi-urban area to determine the relationship between RSSI and link error rate. In their scenario external interference is the main cause for unpredictable link behavior. To model interference effects of directional antennas, the majority of publications [17], [18], [19], [20] have adapted the so called cone (3D) or pie-slice (2D) interference model shown in Figure 2. However, this model does not consider any sideor back-lobes of real-world antennas. The authors in [21] comprehensively show that the pie-slice model exaggerate the throughout attained in ad-hoc networks.

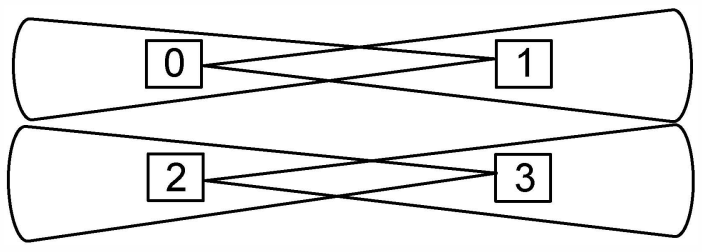

Fig. 2: Pie-slice model of interference. A transmission from 0 to 1 does not interfere with a transmission from 2 to 3 [17].

\section{METHODOLOGY}

Reliable signal measurement is a challenging task, especially when multiple transmitters are present [22]. It is difficult to distinguish between signals at the receiver to determine that a certain transmitter has caused an interference. To overcome this issue and gain insight in the origin of interferences, we conducted a simulation based study.

The simulations in this work are based on a recent extension of ns-3 in version 3.26 to simulate LTE and WiFi coexistence in unlicensed spectrum, Licensed-Assisted Access (LAA) [23]. In their work, the authors developed a new module called SpectrumWiFiPhy. Compared to the previous WiFi model YANS, SpectrumWiFiPhy has the ability to simulate WiFi in combination with other wireless technologies on the same channel. It uses ns-3's build-in spectrum framework which allows a fine-grained frequency decomposition of the signal. For our work, the most desired feature of the SpectrumWiFiPhy module is the possibility to use directional antennas. Only a small extension to the originally published code was needed since the spectrum framework already possesses the ability for directional antenna communication.

After initial tests, we found that the current antenna modules in ns-3 version 3.26 do not account for side- or back-lobes. Therefore, we implemented a class which is able to load and use radiation pattern provided by various antenna vendors. This FileAntenna module use the ".ant" v3 data format. This format is a simple text file with 720 lines representing the antenna gain in a one degree granularity for the azimuth and elevation plane alike. For very long links, this one degree granularity leads to unwanted steps in the Received Signal Strength (RSS). Therefore, we conducted a linear approximation between each degree to provide a smoother transition.

The source code of all following ns-3 simulations as well as additional configurations and test have been made available to the research community on our website [24].

Before we provide the reader with our main results, we show a short verification of the FileAntenna module and justify the choice for different simulation parameters in the following.

For all forthcoming tests, we choose the antenna diagram of a Ubiquity PowerBridge M5, a typical device for WiLD deployments in rural areas [2]. A plot of the radiation pattern is shown in Figure 3a. Despite the main-lobe at $0^{\circ}$ with an overall gain of $25 \mathrm{dBi}$, the radiation pattern reveals several side-lobes. On the azimuth plane at: $15^{\circ}, 26^{\circ}, 334^{\circ}$ and $345^{\circ}$. On the elevation plane at: $61^{\circ}, 74^{\circ}, 106^{\circ}$ and $119^{\circ}$.

To verify the FileAntenna module, we simulate a WiFi node with the contemplated directional antenna in the origin of a three dimensional space. We moved a second WiFi node with an omni-directional antenna around this center in two degree circles and at an increasing radius up to $200 \mathrm{~m}$. We use simple UDP traffic and traced the RSS at the SpectrumWiFiPhy module. We conducted this test for the azimuth $(x-y)$ and the elevation plane $(\mathrm{x}-\mathrm{z})$. The results are shown in Figure $3 \mathrm{~b}$. The figure reveals that the FileAntenna module is working as intended. A wide-range of RSSs at different angles correspond to the antenna diagram shown in Figure 3a.

For the forthcoming main simulations, we list the common parameters in Table I. The choice is based on our realworld WiLD links [1] using a linux based operating systems 
TABLE I: Selected simulation parameters.

\begin{tabular}{lll}
\hline Parameter & Choice & Reason \\
\hline Standard & $802.11 \mathrm{n}$ & According to [1] \\
Freq./Width & $5180 / 20 \mathrm{MHz}$ & First U-NII-2e channel \\
MAC-Layer & AdhocWifiMac & According to [1] \\
Station Manager & ConstantRate & $\begin{array}{l}\text { Minstrel is not used for sta- } \\
\text { tionary links [25] }\end{array}$ \\
Data Rate & MCS7 & Maximum SISO rate \\
Control Rate & MCS3 & ACK rate for MCS7 [1] \\
A-MPDU-Size & 8192 Bytes & According to [1] \\
RTS/CTS & Disabled & According to [1] \\
TxPower & 5 dBm & 5 dBm + AntennaGain $=$ \\
& & EIRP, regulated by FCC \\
Simulation Time & $30 \mathrm{~s}$ & Constant output after this \\
& & time $(\Delta<0.1 \%)$ \\
Traffic & UDP, saturated & According to [1] \\
Distance & $1 \mathrm{~km}$ & A short WiLD link \\
Payload Size & 1450 Bytes & According to [1] \\
Routing & IPv4 Static & Avoid routing overhead, fix \\
& & paths \\
Delay Model & ConstantSpeed & Propagation in air \\
Error-Rate Model & Nist & According to [26] \\
Propagation Model & Friis & No obstacles \\
MAC-Layer timings & Distance depended & According to [1] \\
\hline & &
\end{tabular}
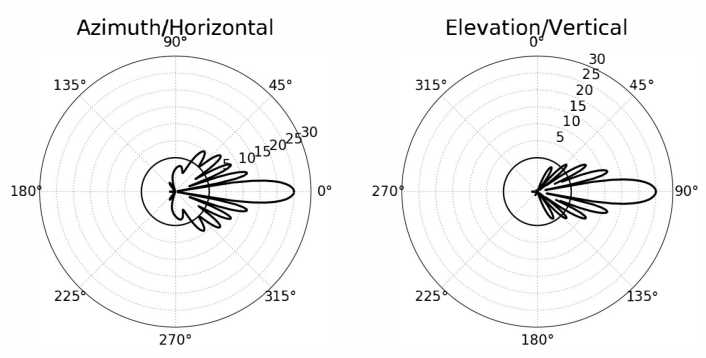

(a) Antenna diagram data-sheet based on .ant file.
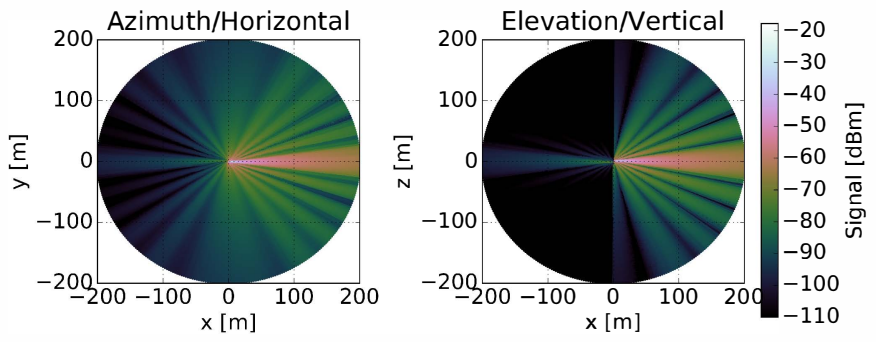

(b) Verification of the Antenna diagramm with ns-3.

Fig. 3: Antenna (Ubiquity PowerBridge M5) used in the simulation. Higher resolution images available online [24].

and Atheros WiFi cards. In fact, we have shown in [27] that ns-3 simulates WiLD links accurately in comparison to a mathematical model and real-world tests. In all upcoming experiments we chose UDP as transport protocol and set the packet rate to saturate the medium at MCS 7. We conducted additional simulations using TCP, due to space limitations the TCP results have been made available as supplemental material on our website [24].

For long-distance links different MAC-Layer timings need to be adapted to ensure a proper working of the DCF. The most prominent value is the so called ACK-Timeout which needs to be set higher to account for the additional distance. The adaption of these timings are described in [1]. All following simulation have been calculated on the high-performance cluster for scientific computing at the Bonn-Rhein-Sieg University described in [28].

\section{RESUlTS}

In this section we present the main results of our work regarding the two interference types of interest. We describe each type separately in the following subsections.

\section{A. Intra-flow}

In this subsection we present our experiment to determine the effects of intra-flow interference when using directional antennas. We placed four nodes in a three-dimensional space as shown in Figure 4a. The node $\mathrm{n} 0$ is placed in the origin, $\mathrm{n} 1$ and $\mathrm{n} 2$ are placed along the $\mathrm{x}$-axis at a distance of $1 \mathrm{~km}$. However, $\mathrm{n} 2$ is placed $1 \mathrm{~m}$ higher than $\mathrm{n} 1$ simulating the case of two nodes on the same pole. Nodes n1 and n2 are interconnected using a wired link with significant more capacity than the WiFi channels. The position of $\mathrm{n} 3$ changes for every experiment, the node moves horizontally along a semi-circle with a radius of $1 \mathrm{~km}$ around the point of $\mathrm{n} 1$ and $\mathrm{n} 2$. We introduce an angle $\alpha$ to reduce the position of n3 onto a single value for better visualization of the results. All directional antennas are perfectly aligned towards their corresponding partner.

We trace the different signals inside the SpectrumWiFiPhy module after the reception. In addition, we added the so called Energy Detection Threshold (EDT) as a horizontal line to each plot. The EDT indicates that a node interprets a signal below this threshold as noise, therefore, the carrier sensing function on that node reports an idle medium. As a notation for interference we use the following: source-node $\rightarrow$ interference-node. A transmission from a source-node to a destination-node can cause collisions at the interference-node. As a notation for UDP throughput we use the following: source-node $\Rightarrow$ destination-node.

In this experiment the following three interferences have been identified to explain the relevant effects:

- $n 0 \rightarrow n 2$. A transmission from $\mathrm{n} 0$ to $\mathrm{n} 1$ interferes at $\mathrm{n} 2$.

- $n 0 \rightarrow n 3$. A transmission from $\mathrm{n} 0$ to $\mathrm{n} 1$ interferes at $\mathrm{n} 3$.

- $n 1 \rightarrow n 2$. A local interference between the two antennas at the same $\mathrm{x}-\mathrm{y}$ position.

1) One stream: Figure $4 \mathrm{c}$ shows the results when transmitting a saturating UDP stream $n 0 \Rightarrow n 3$ via $\mathrm{n} 1$ and $\mathrm{n} 2$ which will be further described in the following.

The $n 1 \rightarrow n 2$ interference is negligible small and therefore not plotted. For all angles $\alpha$, this value is well below $-110 d B$. The reason is the very low Elevation/Vertical (x-z) directivity of the antennas of $\approx-39 \mathrm{dBi}$ at $0^{\circ}$ and $180^{\circ}$ respectively. The interference $n 0 \rightarrow n 2$ and $n 0 \rightarrow n 3$ follow a wave form according to the side-lobes of the antenna. The interference $n 0 \rightarrow n 2$ increases due to the rotation of the antenna of $\mathrm{n} 2$. At $\alpha=0^{\circ}$, the back-lobe of $\mathrm{n} 2$ points towards the interference source at n0 leading to a signal of $\approx-82 \mathrm{dBm}$. At $\alpha=180^{\circ}$, both main-lobes are pointed towards each other leading to a 


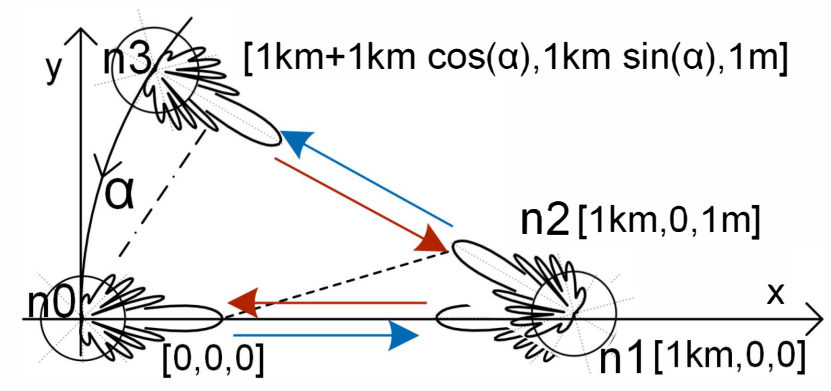

(a) One UDP stream, $n 0 \Rightarrow n 3$ via $\mathrm{n} 1$ and $\mathrm{n} 2$.

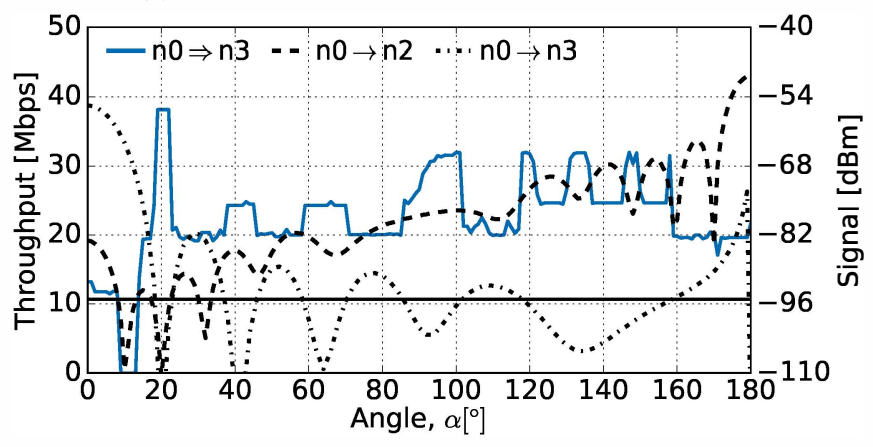

(c) Throughput and interference results for scenario $4 \mathrm{a}$.

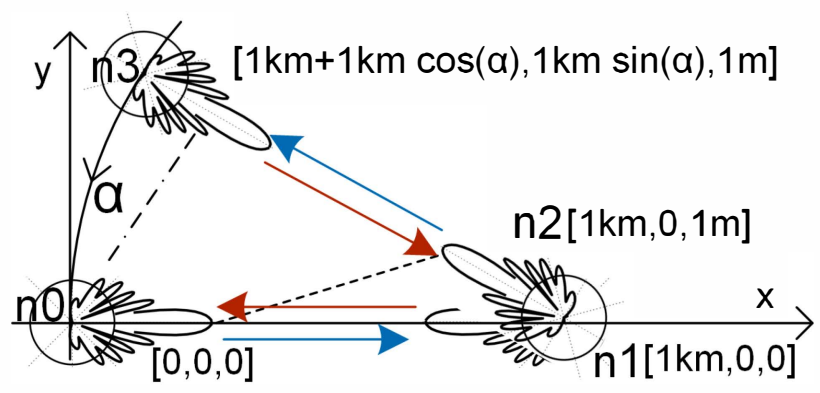

(b) Two UDP streams, $n 0 \Rightarrow n 3$ and $n 3 \Rightarrow n 0$.

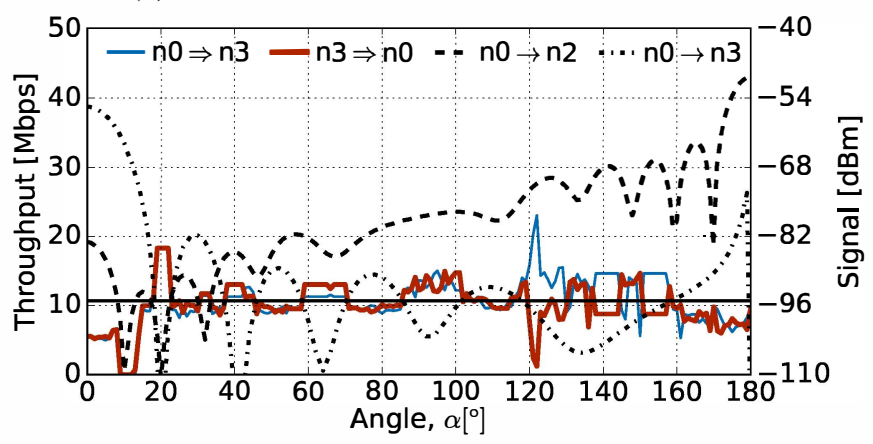

(d) Throughput and interference results for scenario $4 \mathrm{~b}$.

Fig. 4: Simulation scenarios and results for intra-flow interferences.

signal of $\approx-49 d B m$. During the rotation of $n 2$, overall nine local bursts are visible for $n 0 \rightarrow n 2$. These nine different bursts correspond to the side-lobes of the antenna visible in Figure $3 \mathrm{~b}$ - Azimuth. Overall $n 0 \rightarrow n 2$ is for the vast majority of angles above the EDT so that $n 2$ can detect ongoing transmissions between $\mathrm{n} 0$ and $\mathrm{n} 1$ and the carrier sensing function reports a busy medium. At multiple angles, $n 0 \rightarrow n 3$ falls short of the EDT. The carrier sensing function of $n 3$ can not detect an on-going transmission from n0.

Depending on the interference signals, the throughput changes significantly. Several interesting situations occur depending on the angle. In the following we describe four of these situations in more detail:

- At $\alpha \approx 11^{\circ}, n 0 \rightarrow n 2$ falls short of the EDT which means that $\mathrm{n} 2$ is not able to detect a transmission from $\mathrm{n} 0$ to $n 1$. However, $n 3$ is vulnerable to the transmission from n0. Due to the saturating UDP streams, n0 and n2 are constantly transmitting. Collisions at $\mathrm{n} 3$ leads to almost no throughput.

- At $\alpha \approx 21^{\circ}, n 0 \rightarrow n 2$ and $n 0 \rightarrow n 3$ both fall short of the EDT. In this case, there is no interference among both wireless links and the throughput between n0 and n3 peaks.

- At various angles (i.e. at $\alpha \approx 80^{\circ}$ ), $n 0 \rightarrow n 2$ and $n 0 \rightarrow n 3$ are well above the EDT which leads to a nearly constant throughput of $50 \%$.

- The additional peaks of the curve are caused by the chosen error rate model which is described in [26]. For example at $\alpha \approx 40^{\circ}, n 0 \rightarrow n 3$ falls short of the EDT. Even if the DCF at n0 and n2 accidentally choose the same backoff slot to conduct a simultaneous transmission, there is no collision at $\mathrm{n} 3$ which leads to a small throughput increase.

2) Two streams: A similar experiment has been conducted with an additional UDP stream $n 3 \Rightarrow n 0$ via $\mathrm{n} 2$ and $\mathrm{n} 1$. The topology is shown in Figure $4 \mathrm{~b}$ and the results are shown in Figure 4d. As expected, both flows share the available air time, with one flow taking over when the other is on hold due to the DCF/EDT.

\section{B. Inter-flow}

In this subsection we present our experiment to explain the effects of inter-flow interference when using directional antennas. We placed four different nodes in a two-dimensional space as shown in Figure 5a. Two parallel wireless links are formed between $\mathrm{n} 0$ and $\mathrm{n} 1$ as well as $\mathrm{n} 2$ and $\mathrm{n} 3$. All nodes are equipped with directional antennas which are perfectly aligned towards their corresponding partner.

Two different distances need to be considered in this scenario. First, $\Delta x$ is the distance along the wireless links. In this work, we set this distance fixed to $1 \mathrm{~km}$ representing a short WiLD link. The second distance, $\Delta y$ is the spacing between the two wireless links. During the simulation we increment this distance from 5 to $1250 \mathrm{~m}$ in 5 meter steps. To describe the inter-flow interference we trace two different signal strengths.

- $n 1 \rightarrow n 3$. A transmission from $\mathrm{n} 1$ to $\mathrm{n} 0$ interferes at $\mathrm{n} 3$. - $n 1 \rightarrow n 2$. A transmission from $\mathrm{n} 1$ to $\mathrm{n} 0$ interferes at $\mathrm{n} 2$. 


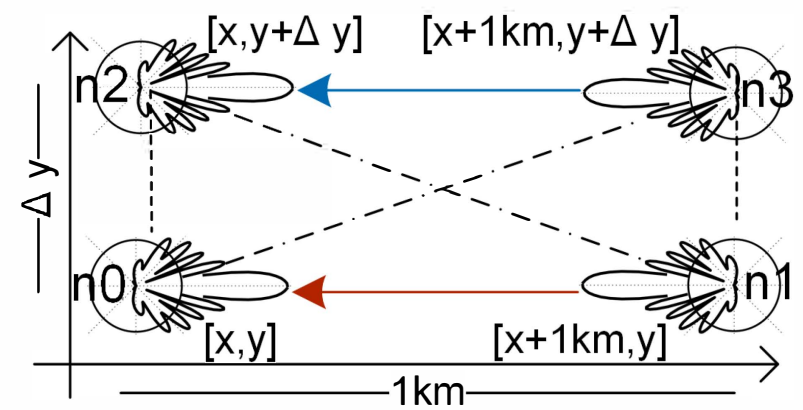

(a) Two UDP streams, $n 1 \Rightarrow n 0$ and $n 3 \Rightarrow n 2$ (same direction).

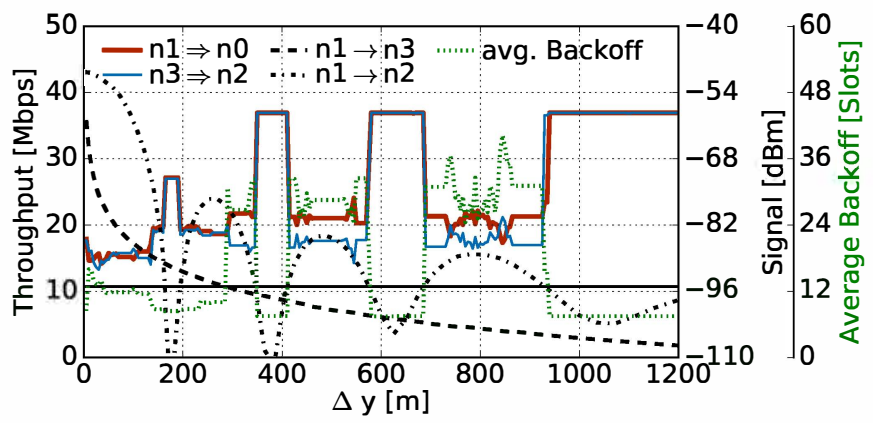

(c) Throughput and interference results for scenario $5 \mathrm{a}$.

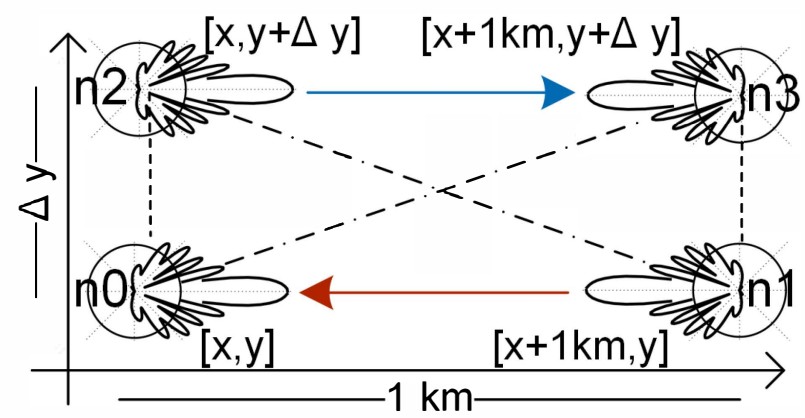

(b) Two UDP streams, $n 1 \Rightarrow n 0$ and $n 2 \Rightarrow n 3$ (different direction).

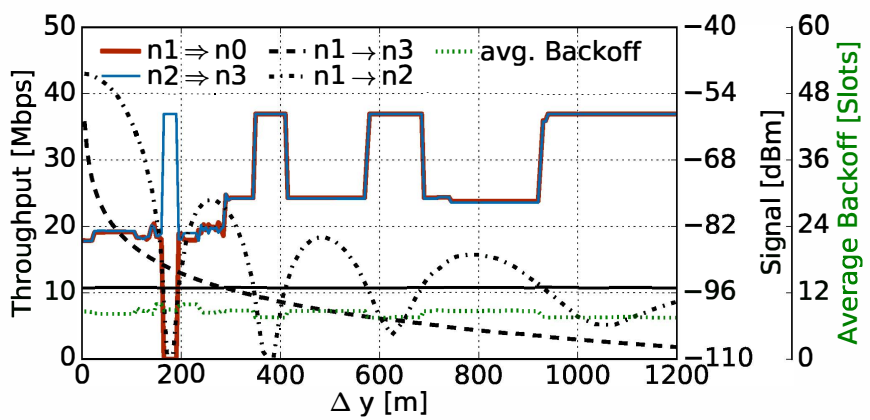

(d) Throughput and interference results for scenario $5 \mathrm{~b}$.

Fig. 5: Simulation scenarios and results for inter-flow interferences.

We saturate both wireless links independently with two different UDP streams. In addition, we conducted two different simulation scenarios depending on the direction of one stream: Either both UDP flows are in the same direction (from $n 1$ to $\mathrm{n} 0$ and $\mathrm{n} 3$ to $\mathrm{n} 2$, Figure 5a) or the UDP flows are transmitted in different directions (from $n 1$ to $n 0$ and $n 2$ to $n 3$, Figure $5 b$ ).

The parallel $n 1 \rightarrow n 3$ interference decreases due to the propagation model. At $\Delta y \approx 300 \mathrm{~m}$ it falls short of the EDT. The form of the $n 1 \rightarrow n 2$ interference results from the side-lobes of the antenna ${ }^{1}$. The results also reveal that the signal strength of the side-lobes decreases in accordance to the radiation pattern and the increased distance along the $n 1 \rightarrow n 3$ path. In addition, an increased $\Delta y$ increases the width of the burst, leading to more space where other stations are prone to interference.

1) Same direction: First we focus on the same direction case shown in Figure 5c. Again, the results can be divided into four situations depending on $\Delta y$ and the resulting interference:

- At $\Delta y<300 m$, the $n 1 \rightarrow n 3$ interference is above the EDT, therefore, the DCF is able to coordinate the transmission of the packets and both links share equal time on the medium.

- At $\Delta y \approx 180 m, n 1 \rightarrow n 3$ is above the EDT but $n 1 \rightarrow n 2$ has fallen short. Even if the DCF at $\mathrm{n} 3$ and $\mathrm{n} 1$ choose the same backoff slot to conduct a simultaneous

\footnotetext{
${ }^{1}$ This fact can mathematically validated by calculating the corresponding angle between the two nodes $\left(\beta=\arctan \left(\frac{\Delta y}{1 k}\right)\right)$. For example, the first burst occurs at $268 \mathrm{~m}$ which corresponds to the first side-lobe at $15^{\circ}$.
}

transmission, there is no collision at $\mathrm{n} 0$ and $\mathrm{n} 2$ which leads to a small throughput increase.

- At $\Delta y \approx 385-405 m$ or $\Delta y \approx 570-690 m, n 1 \rightarrow n 3$ and $n 1 \rightarrow n 2$ fall short of the EDT. In this case both wireless links are independent and the throughput on both links nearly doubles.

- At $\Delta y \approx 405-575 m$ or $\Delta y \approx 690-940 m, n 1 \rightarrow n 3$ can not be detected but $n 1 \rightarrow n 2$ is still present. The side-lobes of the antenna lead to interference at $\mathrm{n} 1$ and multiple collisions occur. We validated these collisions using the average number of backoff slots of the DCF tracked on $\mathrm{n} 1$ shown in Figure 5c. Compared to other distances, the average backoff slots are significantly higher. This effect occurs since the carrier sensing function of $n 1$ is unable to track an ongoing transmission on $n 3$ since the $n 1 \rightarrow n 3$ interference has fallen short of the EDT. Therefore, both carrier sensing function constantly report an idle medium and both $\mathrm{n} 1$ and $\mathrm{n} 3$ start to transmit. Due the $n 1 \rightarrow n 3$ and $n 2 \rightarrow n 1$ interference collisions at the receivers occur. The transmissions are damaged and the number of backoff slots increases up to its maximum of 1023 slots. However, this increased backoff time is also the reason that a data transmission is still possible. If $n 1$ is in a DCF state with an increased backoff time, it is possible for $\mathrm{n} 3$ to conduct a successful data transmission between two attempts of $n 1$.

2) Different directions: Figure 5b shows the same experiment, but with two UDP streams transmitting towards different directions. The average backoff tracing reveals that 
the DCF works in this experiment for all distances. The transmitting nodes $\mathrm{n} 1$ and $\mathrm{n} 2$ are able to track each others transmissions during the previously more complex situation between $\Delta y \approx 405-575 \mathrm{~m}$ and $\Delta y \approx 690-940 \mathrm{~m}$. The most remarkable difference occurs at $\Delta y \approx 180 \mathrm{~m}$ where only one stream occupies significantly more bandwidth.

\section{CONCLUSION AND FUTURE WORK}

In this work we have presented our initial work for interference among long-distance WiFi links with directional antennas. We have successfully implemented an antenna model which has the ability to load real-world radiation patterns in the widely-used ns-3 simulation framework. We selected two interference cases which typically occur in the topology of larger networks and conducted a detailed analysis of the results.

The results reveal that radiation patterns of real-world directional antennas have a significant impact on the throughput. Especially the side-lobes of the directional antennas lead to interference situations which demand a detailed investigation. The widely used cone model would lead to significantly different results not reflecting real-world build-ups.

The results in this work depend on various parameters which we have chosen to the best of our knowledge due to our experiences with WiLD networks. Different radiation patterns lead to different results and evaluating these in additional usecases seems of interest for network planning. At WiFi protocol level, there are different parameters worth to evaluate, for example the usage of RTS/CTS to avoid certain interference situations resulting from side-lobes. However, especially on long-distance links, the usage of RTS/CTS is expensive in terms of throughput reduction due to the increased ACKTimeout. Our main future work item is the evaluation of different Channel Assignment (CA) algorithms for WiLD networks. For these more complex simulations, important aspects like adjacent channel power leakage, external interferences and more accurate propagation modeling still need to be addressed.

\section{ACKNOWLEDGMENT}

This work has been funded by the Federal Ministry of Education and Research of the Federal Republic of Germany (Foerderkennzeichen 16KIS0332). The authors alone are responsible for the content of this paper.

\section{REFERENCES}

[1] M. Rademacher, "Performance estimation and optimization of the IEEE802.11 MAC layer for long distance point-topoint links," Hochschule Bonn-Rhein-Sieg, Tech. Rep., 2015. [Online]. Available: http://opus.bib.hochschule-bonn-rhein-sieg.de/opus3.3/volltexte/2015/30

[2] Ubiquiti Networks, Inc. (2016). [Online]. Available: https://www.ubnt.com/broadband/technology/

[3] Mimosa Networks, Inc. (2016). [Online]. Available: https://www.mimosa.co/technology/

[4] Cambium Networks, LTD. (2016). [Online]. Available: http://www.cambiumnetworks.com/resources/type/white-paper/

[5] "Specific requirements Part 11: Wireless LAN Medium Access Control (MAC) and Physical Layer (PHY) Specification," IEEE P802.11REVmc/D4.0, January 2015 (Revision of IEEE Std 802.11-2012), pp. 1-3730, Feb 2015.
[6] "Improving spatial reuse in multihop wireless networks - A survey," IEEE Commun. Surveys Tuts., vol. 11, no. 3, pp. 71-91, 2009.

[7] nsnam. (2016). [Online]. Available: https://www.nsnam.org/

[8] F. Shzu-Juraschek, "Distributed channel assignment for multi-radio wireless mesh networks," PhD Thesis, Humboldt University of Berlin, 2014.

[9] R. R. Choudhury, X. Yang, N. H. Vaidya, and R. Ramanathan, "Using directional antennas for medium access control in ad hoc networks," in Proc. 8th Annu. Int. Conf. on mobile computing and networking. ACM Press, 2002, p. 59.

[10] M. Takai, J. Martin, R. Bagrodia, and A. Ren, "Directional virtual carrier sensing for directional antennas in mobile ad hoc networks," in Proc. 8th Int. symposium on Mobile Ad Hoc Networking and Comput. ACM Press, 2002, p. 183.

[11] R. Roy Choudhury and N. H. Vaidya, "Performance of ad hoc routing using directional antennas," Ad Hoc Networks, vol. 3, no. 2, pp. 157173,2005

[12] T. Korakis, G. Jakllari, and L. Tassiulas, "CDR-MAC: A Protocol for Full Exploitation of Directional Antennas in Ad Hoc Wireless Networks," IEEE Trans. on Mobile Comput., vol. 7, no. 2, pp. 145155,2008

[13] H.-N. Dai, K.-W. Ng, M. Li, and M.-Y. Wu, "An overview of using directional antennas in wireless networks," Int. J. of Commun. Systems, vol. 26, no. 4, pp. 413-448, Apr 2013.

[14] V. Angelakis, N. Kossifidis, S. Papadakis, V. Siris, and A. Traganitis, "The effect of using directional antennas on adjacent channel interference in 802.11a: Modeling and experience with an outdoors testbed," in Proc. 6th Int. Symposium on Modeling and Optimization. IEEE, 2008.

[15] E. Z. Tragos, A. Fragkiadakis, I. Askoxylakis, and V. a. Siris, "The impact of interference on the performance of a multi-path metropolitan wireless mesh network," Proc. IEEE Symposium on Computers and Commun., pp. 199-204, 2011.

[16] B. Raman, K. Chebrolu, D. Gokhale, and S. Sen, "On the Feasibility of the Link Abstraction in Wireless Mesh Networks," IEEE/ACM Trans. Netw., vol. 17, no. 2, pp. 528-541, apr 2009.

[17] S. M. Das, H. Pucha, D. Koutsonikolas, Y. C. Hu, and D. Peroulis, "DMesh: Incorporating practical directional antennas in multichannel wireless mesh networks," IEEE J. Sel. Areas Commun., vol. 24, no. 11, pp. 2028-2039, 2006.

[18] W. Zhou, X. Chen, and D. Qiao, "Practical Routing and Channel Assignment Scheme for Mesh Networks with Directional Antennas," in IEEE Int. Conf. on Commun. IEEE, 2008, pp. 3181-3187.

[19] Q. Liu, X. Jia, and Y. Zhou, "Topology control for multi-channel multi-radio wireless mesh networks using directional antennas," Wireless Networks, vol. 17, no. 1, pp. 41-51, Jan 2011.

[20] N. Sadeghianpour, T. C. Chuah, and S. W. Tan, "Joint channel assignment and routing in multiradio multichannel wireless mesh networks with directional antennas," Int. Journal of Commun. Systems, vol. 28, no. 9, pp. 1521-1536, Jun 2015.

[21] M. M. Carvalho and J. J. Garcia-Luna-Aceves, "Modeling Wireless Ad Hoc Networks with Directional Antennas," in Proc. IEEE INFOCOM. IEEE, 2006, pp. 1-12.

[22] M. Rademacher and M. Kessel, "An Empirical Evaluation of the Received Signal Strength Indicator for fixed outdoor 802.11 links," VDE ITG-Fachbericht Mobilkommunikation, vol. 20, pp. 62-66, 2015.

[23] L. Giupponi, T. Henderson, B. Bojovic, and M. Miozzo, "Simulating LTE and Wi-Fi Coexistence in Unlicensed Spectrum with ns-3," apr 2016. [Online]. Available: http://arxiv.org/abs/1604.06826

[24] Michael Rademacher. (2016) Multimedia communication laboratory. [Online]. Available: http://www.mc-lab.de/

[25] M. Kretschmer, T. Horstmann, P. Batroff, M. Rademacher, and G. Ghinea, "Link calibration and property estimation in self-managed wireless back-haul networks," in 18th Asia-Pacific Conf. on Commun. IEEE, Oct 2012, pp. 232-237.

[26] G. Pei and T. R. Henderson, "Validation of OFDM error rate model in ns-3," Boeing Research Technology, pp. 1-15, 2010.

[27] M. Rademacher, M. Chauchet, and K. Jonas, "A Token-Based MAC For Long-Distance IEEE802.11 Point-To-Point Links," VDE ITGFachbericht Mobilkommunikation, 2016.

[28] J. Razzaq, R. Berrendorf, J. P. Ecker, S. E. Scholl, S. Augustin, and F. Mannuss, "Performance Characterization of Multiprocessors and Accelerators Using," International Journal On Advances in Systems and Measurements, vol. 9, no. 1, pp. 77-90, 2016. 